\title{
Standard Model Low Mass Higgs Search at CDF
}

\author{
Yoshikazu Nagai ${ }^{* \dagger}$ \\ University of Tsukuba \\ E-mail: nagai@hep.px.tsukuba.ac.jp
}

\begin{abstract}
This paper reports searches for the low mass Standard Model Higgs boson through the decay channel $H \rightarrow b \bar{b}$. The searches use data up to an integrated luminosity of $5.7 \mathrm{fb}^{-1}$ accumulated in the CDF II detector. To achieve maximal sensitivity on the Higgs boson discovery, we analyze many channels: $W H \rightarrow \ell v b \bar{b}, Z H \rightarrow v v b \bar{b}, Z H \rightarrow \ell \ell b \bar{b}$, and $W H / Z H / V B F \rightarrow j j b \bar{b}$. To further improve the sensitivity of the analyses, discrimination between the Higgs boson signal and the large background is improved by employing sophisticated multivariate techniques. The number of observed events and resulting multivariate output distributions are consistent with the Standard Model expectations, and we set 95\% confidence level (C.L.) upper limits on cross section of each process.
\end{abstract}

35th International Conference of High Energy Physics - ICHEP2010,

July 22-28, 2010

Paris France

*Speaker.

$\dagger$ On behalf of the CDF Collaboration 


\section{Introduction}

The success of the Standard Model (SM) in explaining and predicting experimental data provides strong motivation for the existence of a neutral Higgs boson. Current electroweak fits combined with direct searches from LEP2 and Tevatron indicate the mass of the Higgs boson is less than $158 \mathrm{GeV} / c^{2}$ at $95 \%$ C.L. [1, 2].

In proton-antiproton collisions of $\sqrt{s}=1.96 \mathrm{TeV}$ at the Tevatron, the SM Higgs boson may be produced through gluon fusion $(g g \rightarrow H$ ), in association with a vector boson $(q \bar{q} \rightarrow W H$ or $Z H$ ), and through vector boson fusion (VBF) $(q \bar{q} \rightarrow q H \bar{q})$. For low Higgs masses (below $135 \mathrm{GeV} / c^{2}$ ), the dominant decay mode is $H \rightarrow b \bar{b}$. In this report, we present analyses using $H \rightarrow b \bar{b}$ at the $\mathrm{CDF}: p \bar{p} \rightarrow W H \rightarrow \ell v b \bar{b}$ [3, 4], $p \bar{p} \rightarrow Z H \rightarrow v v b \bar{b}$ [5], $p \bar{p} \rightarrow Z H \rightarrow \ell \ell b \bar{b}$ [6], and the all hadronic channel $(W H / Z H / V B F \rightarrow j j b \bar{b})[7]$.

\section{Bottom Quark Tagging Algorithms}

To greatly reduce the backgrounds to these Higgs boson searches, we require that at least one or both two jets from the Higgs boson decay in the event be identified as originating from a $b$-quark. To be " $b$-tagged" the jets from a $b$-quark, we employ three algorithms: the secondary vertex tagging algorithm which identifies $b$-quarks by fitting tracks displaced from the primary vertex [8], the jet probability tagging algorithm which identifies $b$-quarks by requiring a low probability that all tracks contained in a jet originated from the primary vertex based on the track impact parameters [9], and the artificial neural network (NN) tagging algorithm that identifies $b$-quarks by combining the information of displaced vertex, displaced tracks and soft muon [3].

\section{Higgs Search in $p \bar{p} \rightarrow W H \rightarrow \ell v b \bar{b}$}

We select $W H \rightarrow \ell v b \bar{b}$ candidate events with two high- $E_{T}$ jets, large transverse missing energy, and exactly one lepton (electron or muon) from the $W$ boson decays. We present two latest results that employ different strategy on the final signal-to-background separation. One analysis uses the Bayesian Neural Network technique (BNN), and another uses the Matrix Element method (ME). The expected and observed limit for a Higgs boson mass of $115 \mathrm{GeV} / c^{2}$ for these channels are shown in Table 1. The distributions used to set the upper limit are shown in Figure 1(a)(b).

\section{Higgs Search in $p \bar{p} \rightarrow Z H \rightarrow v v b \bar{b}$}

We select $Z H \rightarrow v v b \bar{b}$ candidate events with two high- $E_{T}$ jets and large transverse missing energy. The additional acceptance from the $W H \rightarrow \ell v b \bar{b}$ (lepton is escaped from the detector acceptance) is also taken account. To remove overwhelming QCD background, we develop the method to effectively reduce QCD background using a NN technique. The final discrimination between signal and background is achieved by employing another $\mathrm{NN}$ discriminant. The expected and observed limit for a Higgs boson mass of $115 \mathrm{GeV} / \mathrm{c}^{2}$ for this channel is shown in Table 1. The distribution used to set the upper limit is shown in Figure 1(c).

\section{Higgs Search in $p \bar{p} \rightarrow Z H \rightarrow \ell \ell b \bar{b}$}

We select $Z H \rightarrow \ell \ell b \bar{b}$ candidate events with two high- $E_{T}$ jets and two high- $p_{T}$ leptons from the $\mathrm{Z}$ boson decays $\left(e^{+} e^{-}\right.$or $\left.\mu^{+} \mu^{-}\right)$. To increase the signal acceptance, we include the new channel by requiring a pair of loose muons, which muons are identified using a NN technique. The final 


\begin{tabular}{c|c|c|c}
\hline Channel & Int. Luminosity $\left(\mathrm{fb}^{-1}\right)$ & Expected/SM & Observed/SM \\
\hline$p \bar{p} \rightarrow W H \rightarrow \ell v b \bar{b}(\mathrm{BNN})$ & 5.7 & 3.5 & 4.5 \\
$p \bar{p} \rightarrow W H \rightarrow \ell v b \bar{b}(\mathrm{ME})$ & 5.6 & 3.5 & 3.6 \\
$p \bar{p} \rightarrow Z H \rightarrow v v b \bar{b}$ & 5.7 & 4.0 & 2.3 \\
$p \bar{p} \rightarrow Z H \rightarrow \ell \ell b \bar{b}$ & 5.7 & 5.5 & 6.0 \\
$p \bar{p} \rightarrow V H \rightarrow j j b \bar{b}$ and $V B F H \rightarrow b \bar{b}$ & 4.0 & 17.8 & 9.1 \\
\hline
\end{tabular}

Table 1: Expected and observed upper limits for a Higgs boson mass of $115 \mathrm{GeV} / c^{2}$ for the five low mass Higgs channels.

discrimination between signal and background is achieved by employing a two dimensional NN discriminant. The expected and observed limit for a Higgs boson mass of $115 \mathrm{GeV} / \mathrm{c}^{2}$ for this channel is shown in Table 1. The distribution used to set the upper limit is shown in Figure 1 (d).

\section{Higgs Search in $p \bar{p} \rightarrow V H \rightarrow j j b \bar{b}$ and $V B F H \rightarrow b \bar{b}$}

We select $p \bar{p} \rightarrow j j b \bar{b}$ Higgs signal candidate events by requiring four or five high- $E_{T}$ jets. The signal process that makes $j j b \bar{b}$ final state could come from both the associated production process with a vector boson and the vector boson fusion production process. By considering the kinematic difference for these two processes, we separate the signal region into two region to maximize search sensitivity. Difficult QCD background modeling is achieved by employing datadriven method. The final discrimination between signal and background is achieved by employing NN discriminant. The expected and observed limit for a Higgs boson mass of $115 \mathrm{GeV} / c^{2}$ for this channel is shown in Table 1. The distribution used to set the upper limit is shown in Figure 1(e).

\section{Conclusions}

We have presented the results of a search for the SM Higgs boson decaying to $b \bar{b}$ final state performed at the CDF experiment at the Tevatron. We observe no significant excess and set $95 \%$ C.L.upper limit on the Higgs production cross section. The results presented at this report are expected to significantly improve the overall Tevatron sensitivity in the low mass region Higgs boson searches [10].

\section{References}

[1] LEP Electroweak Working Group, http: // lepewwg.web.cern.ch/LEPEWWG/

[2] ALEPH, DELPHI, L3, OPAL. The LEP Working Group for Higgs Boson Searches, Phys. Lett $\mathbf{B 5 6 5}$ 61 (2003).

[3] CDF Collaboration, T.Aaltonen et al., CDF Conference Note 10239.

[4] CDF Collaboration, T.Aaltonen et al., CDF Conference Note 10217.

[5] CDF Collaboration, T.Aaltonen et al., CDF Conference Note 10212.

[6] CDF Collaboration, T.Aaltonen et al., CDF Conference Note 10221; CDF Collaboration, T.Aaltonen et al., CDF Conference Note 10235.

[7] CDF Collaboration, T.Aaltonen et al., CDF Conference Note 10010. 

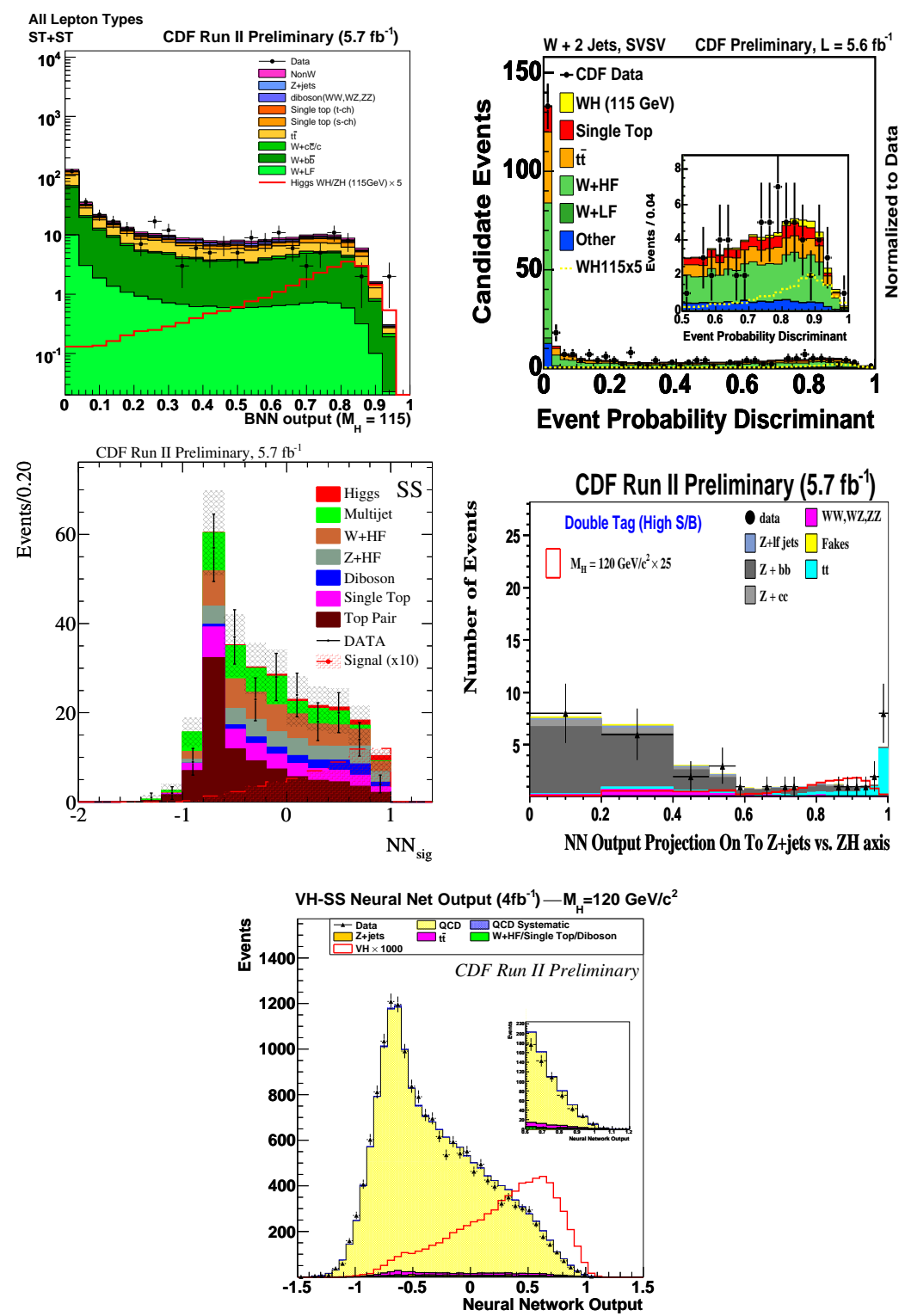

Figure 1: The final discriminant distributions of the $H \rightarrow b \bar{b}$ analyses. From left to right and top to bottom, (a) The distribution of the $p \bar{p} \rightarrow W H \rightarrow \ell v b \bar{b}$ BNN analysis, (b) The distribution of the $p \bar{p} \rightarrow W H \rightarrow \ell v b \bar{b}$ ME analysis, (c) The distribution of the $p \bar{p} \rightarrow Z H \rightarrow v v b \bar{b}$ NN analysis, (d) The distribution of the $p \bar{p} \rightarrow$ $Z H \rightarrow \ell \ell b \bar{b} \mathrm{NN}$ analysis, (e) The distribution of the $p \bar{p} \rightarrow V H \rightarrow j j b \bar{b} \mathrm{NN}$ analysis, respectively.

[8] A. Abulencia et al., Phys. Rev. D71, 072005 (2005).

[9] D. Buskulic, et al. (ALEPH Collaboration), Phys. Lett. B 313, 535 (1993);

F. Abe et al. (CDF Collaboration), Phys. Rev. D 53, 1051 (1996);

A. Affolder et al. (CDF Collaboration), Phys. Rev. D 64, 032002 (2001), Erratum-ibid: Phys. Rev. D

67, 119901 (2003).

[10] CDF Collaboration, T.Aaltonen et al., CDF Conference Note 10241;

D0 Collaboration, V. M. Abazov et al., D0 Conference Note 6096. 